\title{
@s \\ Density functional study of FeS, FeSe, and FeTe: Electronic structure, magnetism, phonons, and superconductivity
}

\author{
Alaska Subedi \\ Department of Physics and Astronomy, University of Tennessee, Knoxville, Tennessee 37996, USA \\ and Materials Science and Technology Division, Oak Ridge National Laboratory, Oak Ridge, Tennessee 37831-6114, USA \\ Lijun Zhang, D. J. Singh, and M. H. Du \\ Materials Science and Technology Division, Oak Ridge National Laboratory, Oak Ridge, Tennessee 37831-6114, USA
}

(Received 29 July 2008; published 13 October 2008)

\begin{abstract}
We report density functional calculations of the electronic structure, Fermi surface, phonon spectrum, magnetism, and electron-phonon coupling for the superconducting phase FeSe, as well as the related compounds $\mathrm{FeS}$ and FeTe. We find that the Fermi-surface structure of these compounds is very similar to that of the Fe-As based superconductors, with cylindrical electron sections at the zone corner, cylindrical hole surface sections, and depending on the compound, other small hole sections at the zone center. As in the Fe-As based materials, these surfaces are separated by a two-dimensional nesting vector at $(\pi, \pi)$. The density of states, nesting, and Fermi-surface size increase, going from FeSe to FeTe. Both FeSe and FeTe show spin-density wave (SDW) ground states, while FeS is close to instability. In a scenario where superconductivity is mediated by spin fluctuations at the SDW nesting vector, the strongest superconductor in this series would be doped FeTe.
\end{abstract}

DOI: 10.1103/PhysRevB.78.134514 PACS number(s): 74.25.Jb, 74.25.Kc, 74.70.Dd, 71.18. +y

\section{INTRODUCTION}

Superconductivity was recently reported in $\alpha$-FeSe ${ }_{1-x}$, with critical temperature $T_{c} \sim 8 \mathrm{~K}$. ${ }^{1}$ This is of interest both because of the fact that $\mathrm{Fe}$ containing superconductors are unusual and because this material shares in common square planar sheets of tetrahedrally coordinated Fe with the Fe-As based high-temperature superconductors. ${ }^{2-7} \alpha$-FeSe occurs in the $\mathrm{PbO}$ structure. This consists, as mentioned, of $\mathrm{Fe}$ square planar sheets with Se atoms forming distorted tetrahedra around the Fe, very similar to the structure of the FeAs planes in $\mathrm{LaFeAsO}, \mathrm{BaFe}_{2} \mathrm{As}_{2}$, and $\mathrm{LiFeAs}$, which are prototypes of the known families of $\mathrm{Fe}$-As based high- $T_{c}$ superconductors. ${ }^{8-10}$

Since the reported $T_{c}=8 \mathrm{~K}$ of doped $\alpha$-FeSe is modest, it is important first of all to establish the relationship between this material and the Fe-As based superconductors. We note that LaNiPO is also a superconductor and shares the crystal structure of LaFeAsO (Refs. 11 and 12) but that it is apparently quite different electronically and can be understood in terms of standard electron-phonon theory, ${ }^{13}$ unlike the Fe-As based phases. ${ }^{14,15}$

Here we report density functional calculations that show $\alpha$-FeSe and the other known Fe based chalcogenides in this structure to be very similar to the Fe-As based superconductors. ${ }^{16}$ In particular the Fermi surface consists of small heavy-hole cylinders near the zone center and lighter compensating electron cylinders around the zone corner. We show that the stoichiometric compounds are either very close to a spin-density wave (SDW) instability (FeS) or have an itinerant spin-density wave instability without doping (FeSe and $\mathrm{FeTe}$ ) similar to the Fe-As superconductors. ${ }^{17}$ We predict that this itinerant nesting driven magnetic state is strongest in FeTe and in addition that FeTe has the largest Fermi surface of the three compounds. Calculations of the electron-phonon coupling show that doped FeSe is not an electron-phonon superconductor, similar to what was found for the Fe-As phases. Within a spin-fluctuation driven picture of superconductivity the results indicate that FeTe with doping is a likely higher-temperature superconductor.

\section{FIRST-PRINCIPLES METHODS AND STRUCTURE}

Our calculations of the electronic structure and magnetic properties were performed within the local-density approximation (LDA) with the general potential linearized augmented plane-wave (LAPW) method, ${ }^{18}$ including local orbitals ${ }^{19}$ similar to our previous calculations for the Fe-As based superconductors. ${ }^{15,16,20}$ We used LAPW spheres of radius $2.1 a_{0}$ for $\mathrm{Fe}, \mathrm{Se}$, and $\mathrm{Te}$, and $1.9 a_{0}$ for $\mathrm{S}$. These compounds occur in a simple tetragonal structure with one internal parameter, $z_{X}$ corresponding to the chalcogen height above the $\mathrm{Fe}$ square plane. The experimental lattice parameters ${ }^{1,21,22}$ were employed and we relaxed the chalcogen height via energy minimization. The structural parameters used and some results are presented in Table I. The electron-phonon coupling and phonon dispersions were on the other hand obtained using linear response, again with the experimental lattice parameters, with the QUANTUM ESPRESSO code ${ }^{23,24}$ within the generalized gradient approximation (GGA) of Perdew, Burke, and Ernzerhof ${ }^{25}$ as described for $\mathrm{LaFeAsO}$ and $\mathrm{LaNiPO}{ }^{13,15}$

\section{ELECTRONIC STRUCTURE, PHONONS, AND MAGNETISM}

Our main results for the electronic structure are given in Figs. 1-3, which show the non-spin-polarized band structures, electronic densities of states, and Fermi surfaces of $\mathrm{FeS}, \mathrm{FeSe}$, and FeTe. The calculated values of $N\left(E_{F}\right)$ are given in Table I. The phonon dispersions of FeSe are shown 
TABLE I. Structural parameters and magnetic properties of PbO-structure Fe $X$. The lattice parameters are from experimental data, while the internal chalcogen structural parameter, $z_{X}$, is from LDA structure minimization. $m_{\mathrm{SDW}}$ is the spin moment within the Fe LAPW sphere (radius 2.1 $a_{0}$ ) for the SDW state and $E_{\mathrm{SDW}}$ is the energy per Fe of this state relative to the non-spin-polarized state in $\mathrm{meV} / \mathrm{Fe} . N\left(E_{F}\right)$ is the density of states at the Fermi energy in the non-spin-polarized band structure in $\mathrm{eV}^{-1}$ on a per Fe both spins basis.

\begin{tabular}{ccccccc}
\hline \hline & $a(\AA)$ & $c(\AA)$ & $z_{X}$ & $N\left(E_{F}\right)$ & $m_{\text {SDW }}\left(\mu_{B}\right)$ & $E_{\text {SDW }}$ \\
\hline $\mathrm{FeS}$ & 3.6735 & 5.0328 & 0.2243 & 1.35 & 0.00 & 0 \\
$\mathrm{FeSe}$ & 3.765 & 5.518 & 0.2343 & 0.95 & 0.65 & 5 \\
$\mathrm{FeTe}$ & 3.8215 & 6.2695 & 0.2496 & 1.83 & 1.28 & 47 \\
\hline \hline
\end{tabular}

in Fig. 4. One interesting feature of the phonon dispersion is that they have little dispersion in $k_{z}$, e.g., are very flat along the tetragonal $\Gamma-Z$ direction. This presumably reflects anionanion repulsion, which leads to long bonds between the FeSe layers. The result is that there may be an easy cleavage plane between the Se ions, which may facilitate preparation of clean surfaces for experiments such as photoelectron spectroscopy.

The phonon density of states (DOS), $G(\omega)$, and electronphonon spectral function, $\alpha^{2} F(\omega)$, are given in Fig. 5. The electron-phonon coupling constant for FeSe as obtained in linear response is $\lambda=0.17$ with $\omega_{\log }=113 \mathrm{~cm}^{-1}$. No superconductivity at any temperature even approaching $1 \mathrm{~K}$ results with these values within standard electron-phonon theory even if very low values of the Coulomb parameter, e.g., $\mu^{*}=0.10$ are used in the Allen-Dynes equation. This is similar to what was found previously for LaFeAsO. ${ }^{14,15}$ Therefore we conclude that $\mathrm{FeSe}$ is not a conventional electron-phonon superconductor.

Turning to the electronic structure we find a strong qualitative similarity between these materials and the FeAs-based superconductors. In particular, we find these to be low carrier density metals with high density of states. This arises from band structures that are closely related to those of the FeAs materials. The chalcogen $p$ states lie well below the Fermi level and are only modestly hybridized with the Fe $d$ states as may be seen from the projected DOS (Fig. 2). Thus the electronic structure near the Fermi energy derives from metallic $\mathrm{Fe}^{2+}$ layers with direct $\mathrm{Fe}-\mathrm{Fe}$ interactions. These are embedded inside a largely ionic background, which imposes a competing tetrahedral crystal field. As in the Fe-As based materials, there is a pseudogap at an electron count of $6 d$ electrons per $\mathrm{Fe}$, and $E_{F}$ lies near the bottom of this pseudogap. We emphasize that this is not the position of a tetrahedral crystal-field gap, which would be at four electrons, and emphasizes the fact that $\mathrm{Fe}$ chalcogen hybridization is not strong compared to the $\mathrm{Fe}-\mathrm{Fe}$ interactions. This explains the similarity of the electronic structure to that of the FeAs-based materials, which were also found to be substantially ionic in similar calculations. ${ }^{16}$

These band structures yield two intersecting elliptical cylindrical electron Fermi surfaces at the zone corner in all three materials. These are compensated by lower velocity hole sections at the zone center-two concentric hole cylinders - and in the case of FeS and FeTe a small closed hole section inside the inner cylinder. This is qualitatively very similar to the FeAs-based materials. It is important to
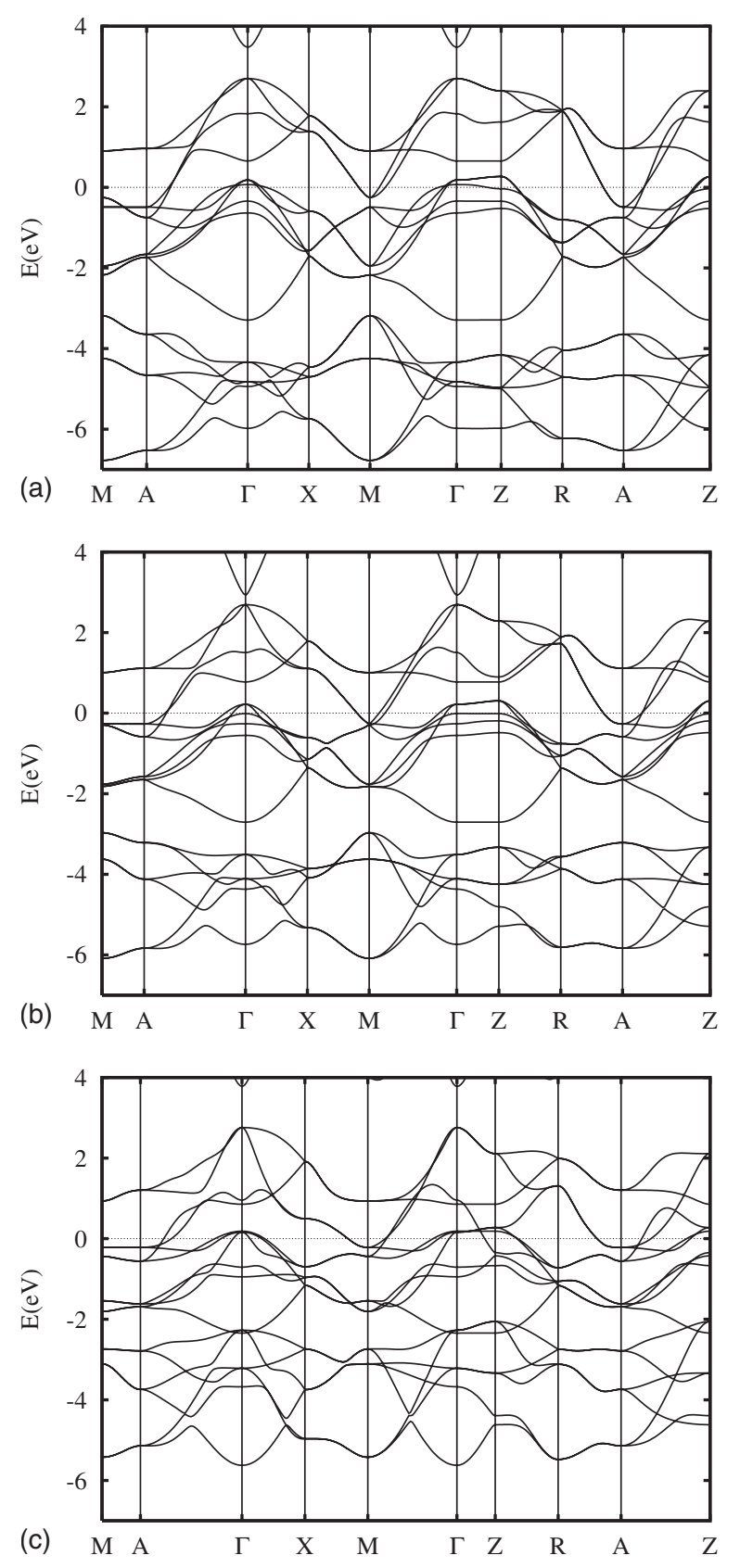

FIG. 1. Band structures of FeS (top), FeSe (middle), and FeTe (bottom) from non-spin-polarized calculations with the LDA relaxed $X$ heights. 

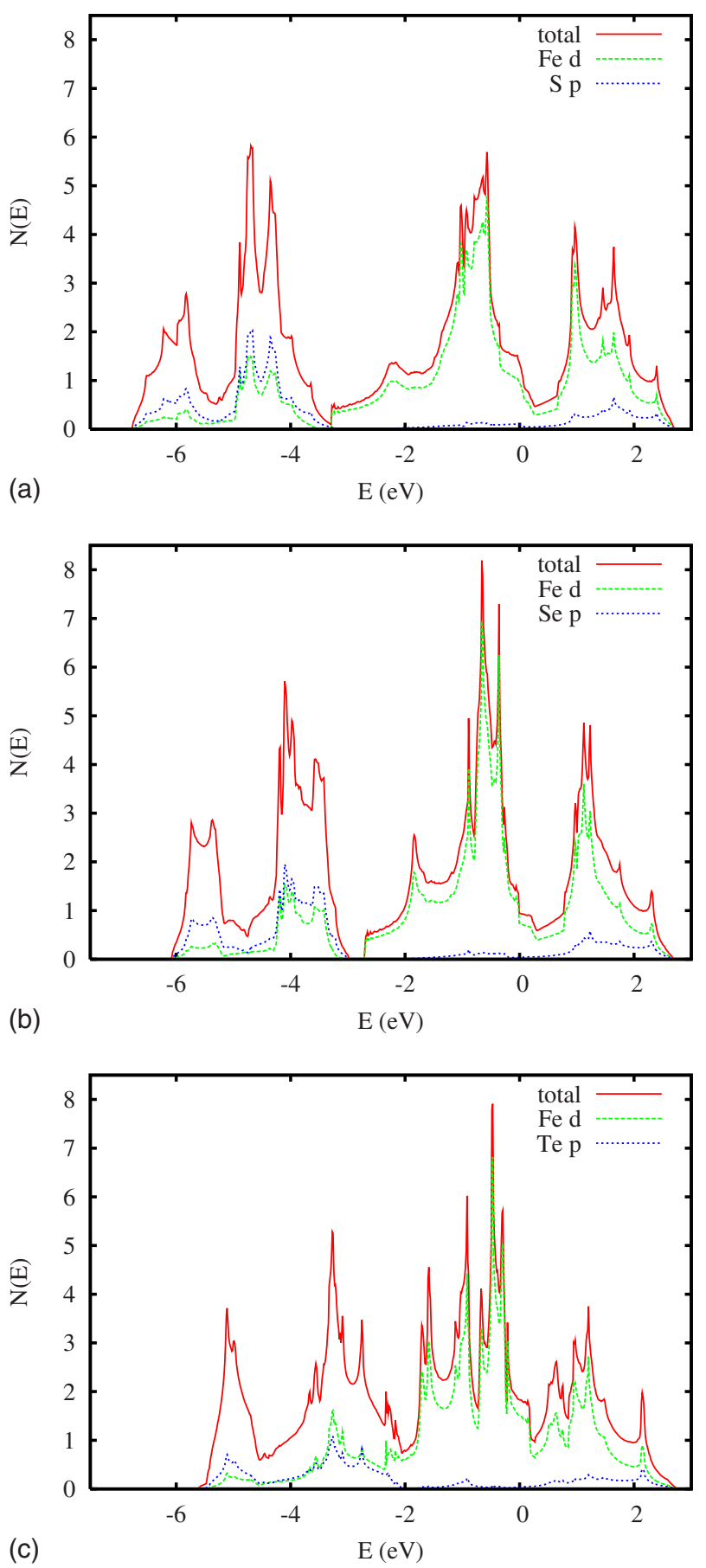

FIG. 2. (Color online) Electronic DOS and projection onto the LAPW Fe and chalcogen spheres indicating the Fe Fe $d$ and chalcogen $p$ contributions for FeS (top), FeSe (middle), and FeTe (bottom) as in Fig. 1.

note that cylinders at the zone center and zone corner, if they are of the same size, would yield strong nesting peaked at the 2D $(\pi, \pi)$ point. This will lead in general to enhanced spin fluctuations at the nesting vector, and if sufficiently strong this will cause a spin-density wave. In fact, an ordered SDW was found both in first-principles calculations and in experimental studies for $\mathrm{LaFeAsO}$ and for many other undoped FeAs-based compounds. ${ }^{15,17,26-31}$ There is a clear competition between the SDW and the superconducting state; super-
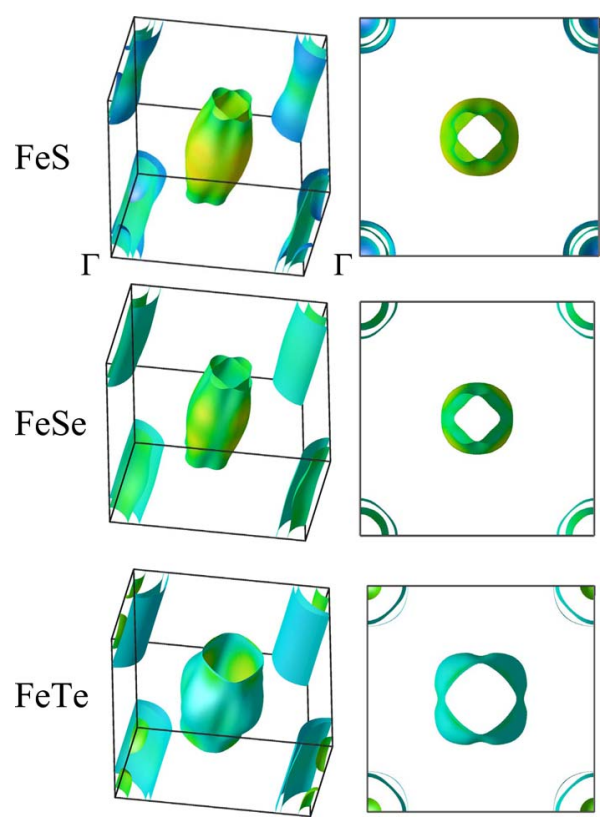

FIG. 3. (Color online) LDA Fermi surface of FeS, FeSe, and FeTe from non-spin-polarized calculations with the LDA relaxed $X$ heights. The corners are $\Gamma$ points.

conducting samples generally do not show the SDW, while samples with the SDW transition, generally do not show superconductivity. The ground state is an antiferromagnetic cell doubled along the [11] in plane direction to yield linear chains of nearest neighbor parallel spin Fe atoms arranged antiferromagnetically although the values of the moments are dependent on details, especially the As height above the $\mathrm{Fe}$ plane. ${ }^{32-37}$

\section{DISCUSSION}

Turning to the trends, the size of the pseudogap is approximately the same in FeSe and FeS but is significantly smaller in FeTe. Specifically, there is a greater overlap between the hole and electron bands in the latter compound. This leads to larger Fermi surfaces. The value of $N\left(E_{F}\right)$ $=1.83 \mathrm{eV}^{-1}$ is also highest in this compound although it is still lower than the $2.62 \mathrm{eV}^{-1}$ that is obtained for $\mathrm{LaFeAsO}$ by the same approach. We used a supercell approach to investigate the SDW with the chalcogen heights fixed to the values calculated by non-spin-polarized energy minimization. We find instabilities for FeSe and FeTe but not for FeS. The spin moments and the energy of the SDW relative to the non-spin-polarized state are given in Table I. As may be seen the SDW is considerably stronger in FeTe than in FeSe, with an energy gain of $47 \mathrm{meV} / \mathrm{Fe}$ and a spin moment of $1.3 \mu_{B}$. The corresponding values for $\mathrm{LaFeAsO}$, calculated in the same way, are $E_{\mathrm{SDW}}=11 \mathrm{meV} / \mathrm{Fe}$ and $m_{\mathrm{SDW}}=1.0 \mu_{B}$ in a $2.1 a_{0} \mathrm{Fe}$ sphere. Besides the SDW we find a borderline ferromagnetic tendency in FeTe, without doping when the SDW is not allowed, similar to LaFeAsO. ${ }^{16}$ We do not find ferromagnetic instabilities in either FeSe or FeS, consistent with the lower values of $N\left(E_{F}\right)$ in those materials. The sensitivity of the moment size to the ordering pattern underscores the 


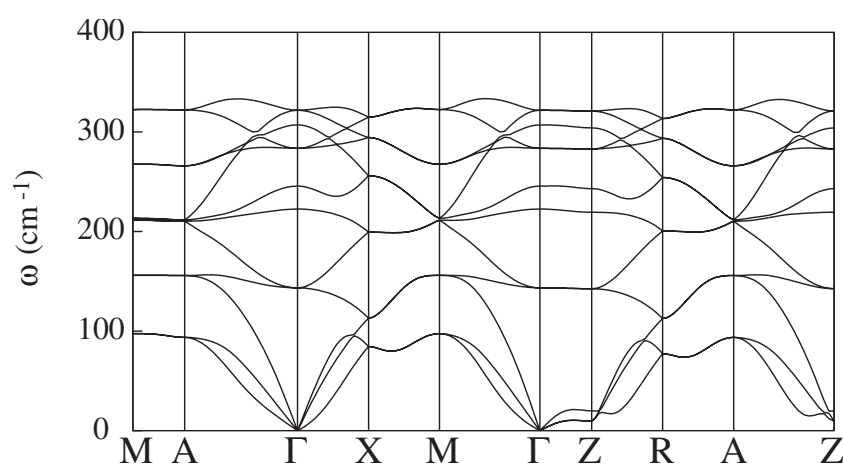

FIG. 4. Calculated GGA phonon dispersions of non-spinpolarized FeSe.

fact that these are itinerant magnetic systems in the LDA, as opposed to local-moment magnets. This means that magnetic ordering is driven by electrons at and near the Fermi surface. On the other hand this is not to say that spin fluctuations are weak in the paramagnetic state above the SDW ordering temperature or in the paramagnetic state as realized by doping. In fact, as noted, several authors have found that the As height in the Fe-As compounds is strongly coupled to magnetism, and so strong spin fluctuations in the paramagnetic state would help rationalize the underestimated As height in non-spin-polarized LDA calculations. ${ }^{32-35,37}$ In fact, there is evidence for strong spin fluctuations in the normal state of the FeAs compounds, e.g., from temperature-dependent resistivity data indicating strong scattering above the SDW ordering temperature (note a drop in resistivity below the SDW ordering temperature even though the carrier density is strongly reduced), ${ }^{38}$ as well as from spectroscopy. ${ }^{39}$ The size of the effects observed implies that these fluctuations should have large amplitudes, and therefore unlike the SDW this should be rather diffuse in q-space, which might make them hard to be directly observed. ${ }^{40}$

We emphasize also that our results in Table I are at the LDA relaxed atomic positions for the non-spin-polarized states. These systems become more magnetic as the chalcogen height is raised. In the case of $\mathrm{FeS}$ a recent refinement is available, and this gives $z_{\mathrm{S}}=0.2602 .{ }^{21}$ This puts the $\mathrm{S}$ ions $0.18 \AA$ higher than that in the LDA structure. With this value we find a stable SDW for FeS, with a moment of $1.2 \mu_{B} / \mathrm{Fe}$. This is the same trend as in the Fe-As based superconducting materials. The result may be taken as an indication that in fact FeS may have a spin-density wave as well and at least that there will be strong spin fluctuations in FeS as well as in other PbO-structure Fe chalcogenides.

As mentioned, cylindrical Fermi-surface sections of equal volume will be nested with nesting vector equal to the separation of the centers of these cylinders. This nesting can be reduced by imperfect matches in shape, three-dimensionality, and size mismatch. Size mismatch can arise both from additional Fermi-surface sections, such as the extra small hole sections obtained in $\mathrm{FeS}$ and FeTe, but not FeSe, and from doping. In particular, electron doping will reduce the size of the hole sections and increase the size of the electron sections consistent with Luttinger's theorem.

The mechanism for superconductivity in the Fe-As based phases has not yet been established. Nonetheless, there are

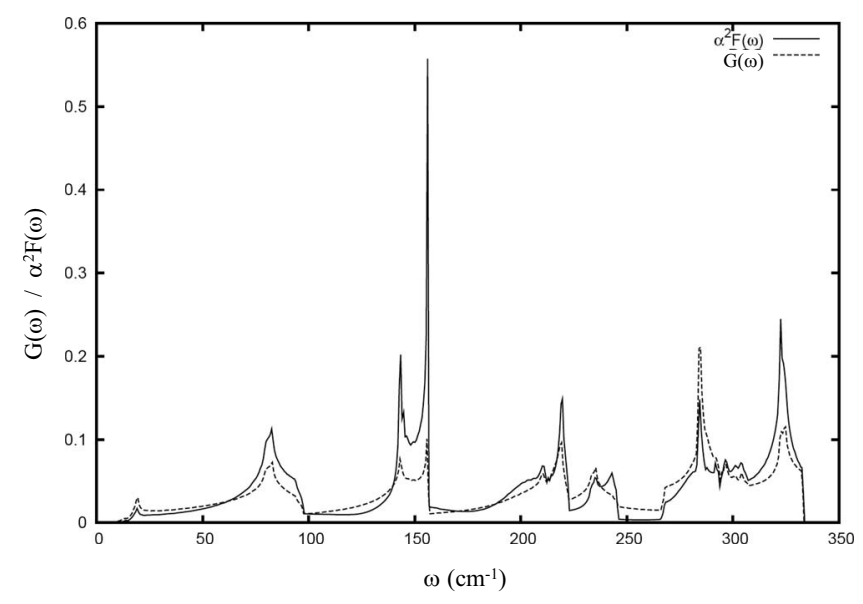

FIG. 5. Calculated GGA phonon density of state $G(\omega)$ and electron-phonon spectral function $\alpha^{2} F(\omega)$ for FeSe.

indications that magnetism is associated with superconductivity. These include the modest electron-phonon couplings in the materials, the proximity to magnetism and the phase diagrams, which show an association between the SDW and superconductivity. We discuss our results within a general spin-fluctuation mediated framework. ${ }^{15,41-43}$ In general an itinerant SDW instability arises from a divergence of the real part of the susceptibility $\chi(\mathbf{q})$ at a specific wave vector $\mathbf{q}$. Superconducting pairing is also associated with the real part of $\chi(\mathbf{q})$ through an integral over the Fermi surface. $\chi(\mathbf{q})$ for q connecting different parts of the Fermi surface can contribute to pairing. As a result, when the Fermi surfaces are small and disconnected as in these Fe based materials, the fluctuations associated with nesting will provide substantial interband pairing between the electron and hole sections, but these will not provide substantial intraband pairing. ${ }^{15,43}$ As the nesting is reduced, e.g., by doping, $\chi(\mathbf{q})$ will spread out while the peak value will be reduced, consistent with the destruction of the SDW. For circular cylinders of radii differing by $\delta q, \chi(\mathbf{q})$ will show a plateau of high $\chi(\mathbf{q})$ around the nesting vector with width $2 \delta q$ (this will persist until the radii differ by a factor of two, at which point the center of the plateau will dip). This means that the two cylinders will still be connected by spin fluctuations associated with their now reduced nesting, and therefore even though the SDW will be suppressed by the reduction in the maximum value of $\chi$, the associated spin fluctuations can still provide superconducting pairing. We emphasize that in this general framework the same parts of the Fermi surface are affected by the SDW and by the superconducting pairing. Thus these two Fermisurface instabilities compete for the same Fermi surface, and therefore there should be at best little coexistence of these two orders.

\section{SUMMARY AND CONCLUSIONS}

We report electronic structures, magnetic properties, and electron-phonon calculations for $\mathrm{Fe} X$, where $X=\mathrm{S}, \mathrm{Se}$, and Te. We find strong similarities to the Fe-As based superconductors, reflecting the ionic nature of the As and chalcogen 
atoms in these compounds. As in arsenides, we find that the electron-phonon coupling cannot explain the superconductivity, and furthermore that these compounds display itinerant magnetism. These results imply a similar superconducting nature for the Fe-As phases and FeSe.

The trend that we find in going from FeSe to FeTe is interesting in this context. In particular we find quite cylindrical Fermi surfaces and an SDW instability in both compounds. However, the strength of the SDW is substantially higher in FeTe as is the size of the Fermi surface. Within the general framework discussed above, FeTe would be expected to have stronger pairing and therefore higher $T_{c}$ than FeSe, assuming that the same mechanism applies in both materials, that both materials can be chemically doped to the optimum carrier density, and that competing instabilities do not prevent superconductivity in that case. It will be of interest to experimentally probe the similarities of FeSe with those of the Fe-As phases and to search for superconductivity in doped FeTe and in the alloy $\mathrm{Fe}(\mathrm{Se}, \mathrm{Te})$.

\section{ACKNOWLEDGMENTS}

We are grateful for the helpful discussions with I. I. Mazin, D. G. Mandrus, and B. C. Sales. This work was supported by the Department of Energy, Division of Materials Sciences and Engineering.
${ }^{1}$ F. C. Hsu, J. Y. Luo, K. W. Yeh, T. K. Chen, T. W. Huang, P. M. Wu, Y. C. Lee, Y. L. Huang, Y. Y. Chu, D. C. Yan, and M. K. $\mathrm{Wu}$, arXiv:0807.2369 (unpublished).

${ }^{2}$ Y. Kamihara, T. Watanabe, M. Hirano, and H. Hosono, J. Am. Chem. Soc. 130, 3296 (2008).

${ }^{3}$ Z. A. Ren, W. Lu, J. Yang, W. Yi, X. L. Shen, Z. C. Li, G. C. Che, X. L. Dong, L. L. Sun, F. Zhou, and Z. X. Zhao, Chin. Phys. Lett. 25, 2215 (2008)

${ }^{4}$ Z. A. Ren, G. C. Che, X. L. Dong, J. Yang, W. Lu, W. Yi, X. L. Shen, Z. C. Li, L. L. Sun, F. Zhou, and Z. X. Zhao, Europhys. Lett. 83, 17002 (2008)

${ }^{5}$ C. Wang, L. Li, S. Chi, Z. Zhu, Z. Ren, Y. Li, Y. Wang, X. Lin, Y. Luo, S. Jiang, X. Xu, G. Cao, and Z. Xu, arXiv:0804.4290 (unpublished)

${ }^{6}$ A. S. Sefat, M. A. McGuire, B. C. Sales, R. Jin, J. Y. Howe, and D. Mandrus, Phys. Rev. B 77, 174503 (2008).

${ }^{7}$ H. Kito, H. Eisaki, and A. Iyo, J. Phys. Soc. Jpn. 77, 063707 (2008).

${ }^{8}$ M. Rotter, M. Tegel, D. Johrendt, I. Schellenberg, W. Hermes, and R. Pottgen, Phys. Rev. B 78, 020503(R) (2008).

${ }^{9}$ M. Rotter, M. Tegel, and D. Johrendt, Phys. Rev. Lett. 101, 107006 (2008).

${ }^{10}$ X. C. Wang, Q. Q. Liu, Y. X. Lv, W. B. Gao, L. X. Yang, R. C. Yu, F. Y. Li, and C. Q. Jin, arXiv:0806.4688 (unpublished).

${ }^{11}$ T. Watanabe, H. Yanagi, T. Kamiya, Y. Kamihara, H. Hiramatsu, M. Hirano, and H. Hosono, Inorg. Chem. 46, 7719 (2007).

${ }^{12}$ M. Tegel, D. Bichler, and D. Jorendt, Solid State Sci. 10, 193 (2008).

${ }^{13}$ A. Subedi, D. J. Singh, and M. H. Du, Phys. Rev. B 78, 060506 (2008).

${ }^{14}$ L. Boeri, O. V. Dolgov, and A. A. Golubov, Phys. Rev. Lett. 101, 026403 (2008).

${ }^{15}$ I. I. Mazin, D. J. Singh, M. D. Johannes, and M. H. Du, Phys. Rev. Lett. 101, 057003 (2008).

${ }^{16}$ D. J. Singh and M. H. Du, Phys. Rev. Lett. 100, 237003 (2008).

${ }^{17}$ C. de la Cruz, Q. Huang, J. W. Lynn, J. Li, W. Ratcliff II, J. L. Zaretsky, H. A. Mook, G. F. Chen, J. L. Luo, N. L. Wang, and P. Dai, Nature (London) 453, 899 (2008).

${ }^{18}$ D. J. Singh and L. Nordstrom, Planewaves, Pseudopotentials and the LAPW Method, 2nd ed. (Springer, Berlin, 2006).

${ }^{19}$ D. Singh, Phys. Rev. B 43, 6388 (1991).

${ }^{20}$ D. J. Singh, arXiv:0807.2643 (unpublished).
${ }^{21}$ A. R. Lennie, S. A. T. Redfern, P. F. Schofield, and D. J. Vaughan, Miner. Mag. 59, 677 (1995).

${ }^{22}$ D. M. Finlayson, D. Greig, J. P. Llewellyn, and T. Smith, Proc. Phys. Soc. London, Sect. B 69, 860 (1956).

${ }^{23} \mathrm{~S}$. Baroni et al., http://www.quantum-espresso.org

${ }^{24}$ Calculations of the phonon spectra and electron-phonon coupling were done using ultrasoft pseudopotentials for $\mathrm{Fe}$, and norm conserving pseudopotentials for Se. We used a 50 Ry basis set cutoff and a high 500 Ry cutoff for the charge-density expansion. This was needed in order to obtain convergence in the linear-response calculations.

${ }^{25}$ J. P. Perdew, K. Burke, and M. Ernzerhof, Phys. Rev. Lett. 77, 3865 (1996).

${ }^{26}$ J. Dong, H. J. Zhang, G. Xu, Z. Li, G. Li, W. Z. Hu, D. Wu, G. F. Chen, X. Dai, J. L. Luo, Z. Fang, and N. L. Wang, Europhys. Lett. 83, 27006 (2008).

${ }^{27}$ G. F. Chen, Z. Li, D. Wu, G. Li, W. Z. Hu, J. Dong, P. Zheng, J. L. Luo, and N. L. Wang, Phys. Rev. Lett. 100, 247002 (2008).

${ }^{28}$ J. Zhao, Q. Huang, C. de la Cruz, S. Li, J. W. Lynn, Y. Chen, M. A. Green, G. F. Chen, G. Li, Z. Li, J. L. Luo, N. L. Wang, and P. Dai, arXiv:0806.2528 (unpublished).

${ }^{29}$ Q. Huang, Y. Qiu, W. Bao, J. W. Lynn, M. A. Green, Y. C. Gasparovic, T. Wu, G. Wu, and X. H. Chen, arXiv:0806.2776 (unpublished).

${ }^{30}$ A. I. Goldman, D. N. Argyriou, B. Ouladdiaf, T. Chatterji, A. Kreyssig, S. Nandi, N. Ni, S. L. Budko, P. C. Canfield, and R. J. McQueeney, arXiv:0807.1525 (unpublished).

${ }^{31}$ Y. Nakai, K. Ishida, Y. Kamihara, M. Hirano, and H. Hosono, J. Phys. Soc. Jpn. 77, 073701 (2008).

${ }^{32}$ T. Yildirim, Phys. Rev. Lett. 101, 057010 (2008).

${ }^{33}$ S. Ishibashi, K. Terakura, and H. Hosono, J. Phys. Soc. Jpn. 77, 053709 (2008)

${ }^{34}$ Z. P. Yin, S. Lebegue, M. J. Han, B. Neal, S. Y. Savrasov, and W. E. Pickett, Phys. Rev. Lett. 101, 047001 (2008).

${ }^{35}$ I. I. Mazin, M. D. Johannes, L. Boeri, K. Koepernik, and D. J. Singh, Phys. Rev. B 78, 085104 (2008).

${ }^{36}$ V. Vildosola, L. Pourovskii, R. Arita, S. Biermann, and A. Georges, Phys. Rev. B 78, 064518 (2008).

${ }^{37}$ T. Yildirim, arXiv:0807.3936 (unpublished).

${ }^{38}$ M. A. McGuire, A. D. Christianson, A. S. Sefat, B. C. Sales, M. D. Lumsden, R. Jin, E. A. Payzant, D. Mandrus, Y. Luan, V. Keppens, V. Varadarajan, J. W. Brill, R. P. Hermann, M. T. 
Sougrati, F. Grandjean, and G. J. Long, arXiv:0806.3878 (unpublished).

${ }^{39}$ F. Bondino, E. Magnano, M. Malvestuto, F. Parmigiani, M. A. McGuire, A. S. Sefat, B. C. Sales, R. Jin, D. Mandrus, E. W. Plummer, D. J. Singh, and N. Mannella, arXiv:0807.3781 (unpublished).

${ }^{40}$ In a system with strong magnetic tendencies ordering will normally occur at high temperature unless there is a large $\mathbf{q}$ space for fluctuations, e.g., due to frustration, itinerant electron physics, or competing exchange interactions. It is difficult to avoid ordering with large amplitude spin fluctuations if they are strongly peaked, e.g., at the SDW nesting vector, or are subject to a simple exchange model that favors a specific ordering pattern, such as a nearest-neighbor antiferromagnetic interaction on the square lattice [with three-dimensional (3D) interactions or magnetoelastic coupling as a present here]. Also, in the case where there is large phase space for fluctuations, local magnetic correlations will be very short range. This does not mean however that the fluctuations will necessarily be fast or only at high energy and in fact near a quantum critical point there will be slow fluctuations that extend to low energy. One experimental signature of such fluctuations besides the magnetostructural effects noted will be via transport data and in particular data that show nearness to a quantum critical point through non-Fermi liquid scalings.

${ }^{41}$ T. Moriya and K. Ueda, Rep. Prog. Phys. 66, 1299 (2003).

${ }^{42}$ P. Monthoux and G. G. Lonzarich, Phys. Rev. B 63, 054529 (2001).

${ }^{43}$ K. Kuroki, S. Onari, R. Arita, H. Usui, Y. Tanaka, H. Kotani, and H. Aoki, Phys. Rev. Lett. 101, 087004 (2008). 